\title{
Review
}

\section{Renal echinococcosis; the parasite, host immune response, diagnosis and management}

\author{
Hossein Yousofi Darani ${ }^{1}$, Rasool Jafari² \\ ${ }^{1}$ Department of Medical Parasitology and Mycology, School of Medicine, Isfahan University of Medical Sciences, \\ Isfahan, Iran \\ ${ }^{2}$ Department of Medical Parasitology and Mycology, School of Medicine, Urmia University of Medical Sciences, \\ Urmia, Iran
}

\begin{abstract}
Renal echinococcosis is a rare parasite-caused disease of humans and animals; it makes up about $4 \%$ of confirmed cases of cystic echinococcosis. It is a zoonotic disease that occurs in the intermediate hosts harboring the larval stage, the hydatid cyst, of Echinococcus spp. The renal involvement is often asymptomatic or with unspecific signs. Its diagnosis is mostly based on imaging technique. Immunodiagnostic tests are not applicable. Furthermore, because the disease is not common, our knowledge about its different aspects is scarce. In this review, the parasite, host immune response, diagnosis, and management of renal echinococcosis are described.
\end{abstract}

Key words: renal echinococcosis; hydatid cyst; immune response; cystic echinococcosis.

J Infect Dev Ctries 2020; 14(5):420-427. doi:10.3855/jidc.11496

(Received 27 March 2019 - Accepted 02 July 2019)

Copyright (C) 2020 Yousofi Darani et al. This is an open-access article distributed under the Creative Commons Attribution License, which permits unrestricted use, distribution, and reproduction in any medium, provided the original work is properly cited.

\section{Introduction}

Cystic echinococcosis (CE) and alveolar echinococcosis (AE) are two important neglected parasite-caused diseases with medical and public health significance [1]. Echinococcosis is a disease of humans and animals caused by different species of Echinococcus. The parasite's life cycle involves carnivorous animals as the definitive hosts and many herbivores and rodents as intermediate hosts. Humans can also be an intermediate host and can harbor hydatid cysts, the larval stage of Echinococcus [2,3]. The adults are a few millimeters long and have 3-5 proglottids; they live in the small intestine of canids and causes no or mild symptoms $[2,4]$. The hydatid cysts, on the other hand, may cause problems in the intermediate hosts, such as economic losses in the livestock industries [5]. In humans, $\mathrm{CE}$ can be serious, depending on the location of the cyst and the resulting complications. However, a considerable number of cases are asymptomatic and diagnosed only accidentally $[4,6]$.

$\mathrm{CE}$ is highly endemic in central Asia, western China, South America, eastern Africa, and in Mediterranean countries. Contact with canids and raising livestock are said to be the main risk factors for the disease. However, active transmission via wildlife has also been reported [7]. Human CE has been eliminated from New Zealand and Tasmania was provisionally CE-free [8], but E. granulosus is still present on the Australian mainland and is found in wild and rural dogs in Tasmania [9]. The elimination and the reduction in the prevalence of $\mathrm{CE}$ have been achieved by long-term efforts towards prevention and control measures, mostly directed to deworming dogs using antihelminth drugs, meat inspection, health education, and surveillance in human and livestock populations. Human CE continues to be a significant but neglected zoonotic disease in numerous countries, especially in resource-poor pastoral areas [8].

The incidence of human alveolar echinococcosis (AE), in contrast, has increased in continental Europe and is considered a major public health issue in some areas of Eurasia [8]. For the past 40 years, AE has been a public health issue in parts of Japan. Studies in China have revealed a considerable incidence of $\mathrm{AE}$ on the Tibetan plateau and interestingly showed that AE, especially in children, is more prevalent than CE in some areas [7]. There are an estimated 18,235 new cases of AE per year globally, of which $91 \%$ are found in China. The majority of these patients live in regions where treatment is not widely available, and consequently, the disease will be fatal. It is estimated that $\mathrm{AE}$ results in a median of 666,434 Disability- 
Adjusted Life Years (DALYs) per annum. This makes $\mathrm{AE}$ one of the very important diseases in particular populations on the Tibetan plateau [10]. The prevalence of E. multilocularis infection in definitive and intermediate hosts in Europe is shown to have increased in the first 15 years of the $21^{\text {st }}$ century. Also, the endemicity of fox infections is more expansive than it was. Urban foxes can be important in the transmission of AE [7]. Human AE cases have been reported in European countries which were previously known to be AE-free. Moreover, the incidence of AE has doubled in the endemic regions of Germany, France, Switzerland, and Austria [7,11-13].

The taxonomy of the genus Echinococcus was controversial for many years. From the oldest descriptions of Echinococcus, the genus exhibits significant variability at the species level with regard to host specificity, antigenicity, morphology, development rate, and transmission cycles. In the latest classification of Echinococcus based on the review by Thompson (2017), it is categorized into ten species, which cause different diseases. They are as follows: $E$. granulosus (causes CE), E. equinus (causes CE), E. ortleppi (causes CE), E. intermedius (causes CE), E. canadensis (causes CE), E. felidis (not defined), E. multilocularis (causes AE), E. shiquicus (causes AE), E. vogeli (causes polycystic echinococcosis, $\mathrm{PE}$ ) and $E$. oligarthra (causes PE) [4].

In the intermediate animals, the microvilli on the surface of the developing metacestode [14], the size of the oncospheres, and the size of the venules and lacteals in different hosts may determine where hydatid cysts will be located among different organs [4]. In primary $\mathrm{CE}$, hydatid cysts may develop in almost any organ. Nearly $80 \%$ of patients with CE have a involvement of a single organ with a solitary cyst localized in the liver or lungs [15]. However, multiple organ involvement is also possible [16]. The liver/lung cyst localization ratio may vary from 2 to 1 in symptomatic patients and up to 9 to 1 in asymptomatic ones. The metacestodes first face the liver, and in some cases, it localizes in there. Larvae that escape the hepatic filter may be carried by the bloodstream to the lungs or less frequently further organs, e.g., to the kidneys, bone, brain [17], heart [18] or muscle tissue [19].

The incidence of human CE is different around the globe, ranging from 0.122 to 15 per 100,000 humans [20]. The incidence of CE in human cases in Iran is estimated at 1.18 to 3 per 100,000 inhabitants (overall incidence: 0.61) [21]. Renal echinococcosis is quite a rare disease compared to the hepatic and pulmonary CE; it makes up about $4 \%$ of confirmed cases of hydatid disease. With regard to the location of the cysts, in $37 \%$ of renal cases, the cysts were located in the upper pole, $34 \%$ were in the lower pole, and 16 in the mediorenal region. The cyst caused kidney damage in $12 \%$ of renal echinococcosis patients. The size of renal hydatid cysts, based on the report from Zmerli et al. (2001) [22], ranges from 2 to $22 \mathrm{~cm}$. In $2 \%$ of these cases, the cyst was also found in the liver, $1 \%$ in the lung, and $0.5 \%$ in the spleen. Furthermore, $25 \%$ of renal hydatid cysts are reported to have ruptured into the urinary tract [22].

The kidney is frequently prone to cyst formation in the human body; cysts are estimated to occur in 5\% of humans. Treatment of simple non-hydatid cysts of the kidney is based on existing complications. Percutaneous aspiration and sclerotherapy are the firstline therapies for these kinds of cysts. Surgical decortication is commonly used for recurring or sizeable symptomatic cysts. Surgery, such as laparoscopy, is highly successful and is linked with minimal morbidity. Retroperitoneal surgery is generally preferred for cysts, especially for renal hydatid cysts, to avoid leakage of hydatid fluid into the peritoneal cavity [23]. Diagnosis of renal echinococcosis can best be achieved by imaging. Serological tests for anti-hydatid cyst antibody are usually give false negative results, although positive reactions have been reported [24].

Most hydatid cysts occur in the right lobe of the liver, perhaps in relation to the anatomy of the liver vein. It may be the same factor affecting renal cysts, which are more often reported from the left kidney than the right one. The latter situation is thought to be influenced by the fact that the left renal artery is shorter [25].

\section{Parasite genetics and renal echinococcosis}

The Echinococcus granulosus complex includes different species, which have different genotypes, namely E. granulosus (G1, G2, and G3 genotypes), $E$. equinus, E. ortleppi, E. intermedius (G6 and G7 genotypes), E. canadensis (G8 and G10), and E. felidis [4]. Among these, E. granulosus is responsible for most of the human infections [26]. There are speculations about the different genotypes/species which may have a different tendency for different organs. For example, Sadjjadi et al. (2013) reported that E. intermedius G6 has a high affinity for the human brain. In their report, all of the eight cerebral hydatid cysts they found belonged to E. intermedius G6 [27]. Few studies have evaluated the genetic characteristics of human isolates of renal hydatid cysts $[26,28,29]$. In these studies, the genotypes isolated from the kidney belonged to $E$. granulosus (G1-G3, strain not defined) [28,29], and $E$. 
granulosus G1 [26]. There have been no comprehensive studies as yet on renal echinococcosis focusing on the parasite genotype, and no evidence has been presented to support the idea that a specific genotype has a high affinity for human kidneys.

\section{Immunology and immunodiagnosis}

Humoral immune response and immunodiagnosis

In $\mathrm{CE}$, an IgG response against hydatid cyst fluid and oncospheral antigens is detectable at two and 11 weeks post-infection, respectively, in the animal model $[1,30]$. The anti-oncospheral antibodies have a significant role in the parasite killing and are essential to produce protective immunity against $E$. granulosus infections in intermediate hosts [31]. It is also suggested that antibody concentrations are low at the beginning of the infection (early stages), while the parasite-killing mechanisms that exist against hydatid cyst may be attributed to antibody-dependent cell-mediated cytotoxicity [1,32].

In chronic $\mathrm{CE}$, elevated antibody levels are detectable mainly for IgG (predominantly IgG4), and $\operatorname{IgE}[33,34,35]$. This increased antibody production is thought to be critical for the advancement of immunological tests [1]. However, from $15 \%$ to nearly $40 \%$ of patients with CE are reported to be antibodynegative [36]. In many antibody-negative patients, certain levels of circulating antigens and circulating immune complexes are detectable [30]. This observation suggests that there is activation and proliferation of B lymphocytes, which may be regulated and inhibited by the parasite antigens, but whether the mechanism/s are directly targeting B cells or working through regulatory mechanisms of $\mathrm{T}$ cells is not known [1].

It has been suggested that immunoglobulins play a role in false immunological test results for echinococcosis. A false-negative immunodiagnosis could be caused by the inactive status of the hydatid cysts, whereas false positives could be caused by previously ruptured cysts, and by cross-reactions among the antigens. Diagnosis of renal echinococcosis can best be achieved by imaging techniques, because of the unreliability of immunodiagnostic tests [24]. Hydatid cyst fluid, protoscoleces, and laminated layerderived antigens were investigated for the detection of CE in humans using western blots; a $20 \mathrm{kDa}$ band of the hydatid cyst fluid appeared to be an appropriate antigen for immunodiagnosis of human CE [37].

Antigen B (AgB) [38] and Antigen 5 (Ag5) [36] are hydatid cyst fluid-derived antigens and are among the most studied antigens for immunodiagnosis of CE.
These antigens are not specific to E. granulosus and can be found in E. multilocularis and E. vogeli infections. Also, fairly high concentrations of anti-Ag5 were detected in the sera of Taenia solium infected patients [39]. The tumoricidal activity of different antigens of hydatid cyst was also evaluated on a range of tumor cells, and some showed promising effects [40-43]; even some common antigens were described between tumors and hydatid cyst [44-46].

The performances of the available serological tests for human CE are not adequate, and the question of which immunological test is better is still the subject of debate among researchers. Despite the fact that several immunodiagnostic tests have been developed, mostly based on hydatid cyst fluid antigens (AgB and Ag5), they are unsatisfactory for the diagnosis of human CE [36]. The situation is even worse for the serological diagnosis of renal $\mathrm{CE}$, because most patients give false negative results $[1,22]$. The Casoni skin test for hydatidosis is reported to be positive in only $21.5 \%$ of patients. Hemagglutination-inhibition (HI) and immunoelectrophoresis tests may become positive in $60 \%$ and $66 \%$ of the patients, respectively [47]. The clinical diagnosis of echinococcosis needs a combination of ultrasonography and immunodiagnosis, which is suggested to be the necessary complementary confirmation [48].

\section{Cellular immune responses}

In the early post-infection phase (establishment phase) of hydatid cyst, marked activation of cellmediated immunity, including some cellular responses and pathological changes, can be measured. Cellular infiltration, including eosinophils, macrophages, neutrophils, and fibrocytes, occurs around the newly located larvae in human infections $[1,49]$. In these early stages of oncosphere establishment, the parasite is very vulnerable to antibody-mediated complementdependent killing [50,51]. In naturally acquired infections, the antibody-mediated immune response may be supplemented by a considerable cellular inflammatory response consisting of macrophages, neutrophils, eosinophils, and lymphocytes in the early four weeks post-infection in sheep. A similar formation of granuloma has been reported from experimental secondary infections with protoscoleces in mice, where immune response-mediated parasite-killing is also documented [51]. However, this usually does not produce a severe inflammatory response, and as they age, the cysts become surrounded by a host-derived fibrous layer that separates the parasite from host tissue [1]. 
As the parasite is vulnerable to killing by immunological responses during the early establishment phase, the existence of degenerating or dead cysts at different later stages of its development suggests that later killing, even though possibly less intense, can arise during the established stage. However, the causes of parasite death in the later phases are not clearly known to be immunologically mediated. The strain/genotype/species of the causative Echinococcus, the intermediate host, and the involved organ can be factors influencing the different levels of parasite development [51-53]. From studies on experimental infections by Echinococcus, it becomes obvious that hydatid cysts from the same genotype of the parasite, developed in the same organ in the same individual host, can display substantial variations in their growth, principally considering their size, viability, and fertility [51].

Two cytokine patterns are described for CD4+ T helper cells in the mouse model, including type 1 helper $\mathrm{T}$ cells (Th1), which release interleukin (IL)-2, tumor necrosis factor-beta (TNF $\beta$ ), and gamma-interferon (IFN $\gamma$ ), and Th2 cells producing IL-6, IL-4, IL-5, and IL10 [54]. Helminth infections are proposed to have immunoregulatory potential. , such that Th2-type responses play a central role in chronic helminthiasis [55]. It is also proposed that a Th2-type response favors the establishment of the larvae of Echinococcus. The Th1-type immune response is regarded as lethal for the parasite in the intermediate hosts. Nevertheless, the situation is not that simple, because often there is a mixed Th1/Th2-type immune response in infected intermediate hosts. Furthermore, regulatory activities by the parasite possibly aim to control the Th1 and Th2 parasite-killing effector mechanisms [56,57]. It has been suggested that in the early phases of experimental infection with protoscoleces, a mixed Th1/Th2-type cytokine response with elevated concentrations of IL10 will develop [58]. Elevated levels of IFN $\gamma$ [59] and IL-5 are also reported in mice inoculated with the protoscoleces. The initial production of IL-4 and IL-10 may be influenced by the parasite as a mechanism to facilitate its establishment [58]. A high level of IL-4, a $\mathrm{Th} 2$ cytokine, was reported in the pericyst of bovine hepatic hydatic cysts [60]. It is not yet clear why the parasite may induce both Th1 and Th2-type cytokine profiles, considering that they typically downregulate each other [1].
The characteristic Th1/Th2-type cytokine profile is also associated with the antigens and the levels of antigen released from the cyst. The AgB released from E. granulosus tilts the cytokine ratios towards an immunopathology-linked Th2 polarization, chiefly in patients with progressive infection. This type of immune modulation may be one of the mechanisms of immune evasion of the parasite from host immune defense [61].

\section{Fibrous layer and immune response}

The initial intensity of host cellular inflammatory reaction varies among hosts and rules the destiny of the developing larvae. A too-intense reaction will cause death and degeneration of the parasite, while in an appropriate intermediate host, the early reaction resolves and leaves a fibrous capsule. The latter circumstance is usual when a steady host-parasite relationship has evolved [4]. The fibrous layer (also called adventitia and pericyst) is a host-derived capsule [51] which encloses hydatid cysts and occurs in the renal cyst as well [62]. It is also important for the local immune response against the parasite $[51,63]$. In large hydatid cysts, its thickness may reach several millimeters and it is made up of several layers adjacent to the normal parenchyma of the organ harboring the cyst $[51,63]$. The pericyst acts to isolate the cyst from the surrounding host tissue and is a consequence of granulomas, which have become fibrotic elements containing collagen fibers. The fibrous layer may also encompass several cell populations such as macrophages and eosinophils. In inactive (dead) cysts, the adventitial layer calcifies, a process regulated by TGF-Beta1[51,64].

\section{Cellular composition of pericyst}

The host reaction site, just behind the fibrous layer of the renal hydatid cyst, is reported to be similar to that of the liver. The palisading macrophages are the first immune cell line after the fibrous elements, and behind them, infiltrations of immune cells appear, mostly $\mathrm{T}$ cells $\left(\mathrm{CD}^{+}\right)$. Among the $\mathrm{T}$ cells, $\mathrm{CD}^{+} \mathrm{T}$ helper cells (Th cells) are predominant in number, followed by $\mathrm{CD}^{+}$cytotoxic $\mathrm{T}$ cells (CTL). Regulatory $\mathrm{T}$ cells (Tregs) are also present in considerable numbers, yet natural killer cells (NK) are rare in the host reaction site of renal hydatid cysts. B cells are present in large numbers, which may be aggregated as granulomas in different areas of the host reaction site, surrounded by $\mathrm{T}$ cells. Eosinophils are also present in large numbers, scattered around the host reaction site [65]. 


\section{Signs and symptoms}

Most patients with renal echinococcosis are asymptomatic or have nonspecific symptoms, and the cyst/s is discovered during screening for other purposes. Reported symptoms include a constant dull pain in the flank, nausea [66], lumbar or abdominal mass similar to a tumor, subcostal pain, abdominal distension, vomiting, and hydatiduria [22]. The reported prevalence of clinical symptoms is as follows: lumbar pain $(80 \%$ of patients), a palpable mass $(25 \%$ of patients), fever (10\% of patients), and nausea and vomiting (10\% of patients). Eosinophilia $(30 \%$ of patients), pyuria ( $25 \%$ of the patients), and hematuria ( $20 \%$ of the patients) may also be seen in patients with renal hydatid cyst. Furthermore, hydatiduria is rare but happens in the case of communicating cysts [47].

\section{Imaging}

Radiography

In radiography, a soft-tissue mass or spherical calcifications in the kidney region may represent a renal hydatid cyst [67]. Caliceal distortion is the major finding during excretory urography, followed by caliectasis and kidney function loss [22].

\section{Sonography}

The typical ultrasound finding is anechoic lesions with well-distinguished borders. Based on the classification by the World Health Organization Informal Working Group on Echinococcosis (WHOIWGE), the ultrasound futures of hepatic CE are sorted as CE1 to CE5. CE1 is characterized by round to oval, unilocular, echo-free cysts, regularly double-walled. Free-floating protoscolices can cause a "snowflake effect" when the patient's position is shifted. In CE2 category, the daughter cysts are observable as "wheel spokes" or a "honeycomb" pattern surrounded by a mother cyst. CE1 and CE2 are indicative of active cysts. $\mathrm{CE} 3$ (CE3a/CE3b) indicates the transitional stage of the cysts, yet activity is also possible. In this stage (CE3), the laminated layer of the cyst's inner side breaks up, showing a degenerative process, and in this stage, the "water lily" sign can be noticed. CE4 corresponds to heterogeneous structures inside the cysts with the look of a "ball of wool." In the CE5, a bow-like calcified thick cyst wall results in a conical ultrasound shadow. In the CE5 category, there are no visible protoscolices. In the CE4 and CE5 stages, the cysts are inactive $[15,68]$.

The ultrasound observation of a layer of liquid material between the layers with no posterior echoes inside and a tumor-like mass, called the "spiral sign," may help to differentiate inactive cysts from renal tumors [67,69]. Moreover, the existence of daughter cyst/s and echoic layer remains, co-existence of hydatid cysts in other organs [67], and a positive serology test [66] favor renal hydatidosis. In inactive lesions, ringlike calcification also appears in the adventitial layer of the hydatid cysts [67].

\section{Computed tomography}

A cyst with a thick or calcified membrane, a multiloculated tumor-like mass with heterogeneous internal density, a unilocular mass with a detached wall, and daughter cyst/s with lower density compared to the maternal matrix are typical findings for renal echinococcosis using standard computed tomography (CT scan). The cyst membrane in CE1 can be defined better using a contrast-enhanced CT scan compared with ultrasound $[67,70]$. Finding daughter cyst/s on CT scan helps to discriminate hydatid cysts from kidney abscesses. CT scan can also help in the diagnosis of inactive hydatid cysts, which looks similar to pseudotumor. This technique can also reveal other organ involvement for hydatid cysts [67].

\section{Management}

Surgery

The optimal treatment for renal echinococcosis is surgery. Surgical management of kidney hydatid cyst can vary and requires pericystectomy or nephrectomy, depending upon the remaining functional parenchyma. Leakage from the cyst should be avoided, because it can cause recurrence or severe anaphylactic shock. Renal hydatid cyst is rare, but it is a quite dangerous disease if not diagnosed precisely [66]. With the surgical approach, either a conservative or a radical cystectomy can be done, depending on the nature of the cyst and the remaining non-damaged renal tissue. The empty cavity that remains after cystectomy is cup-shaped and can be improved by U-stitches applied to the two kidney valves; for a few days a catheter should be placed between them [22]. Nephrectomy is required for about $25 \%$ of renal echinococcosis cases but is suggested only for destroyed kidneys as a result of old, infected, and open cysts. Recently, percutaneous treatment of renal hydatid cyst has been presumed to be safe, but the reported experience is still inadequate [22,71-73]. It is recommended not to treat cyst lesions (CL) until their parasitic nature is confirmed [15]. The heterogeneous appearance of inactive cysts should be distinguished from other lesions like abscesses, infected renal cysts, and tumors [67,73]. Rarely there is operative or 
postoperative mortality after cystectomy, and most patients will be cured and will live well [47].

\section{Chemotherapy}

Chemotherapy with albendazole as adjuvant therapy may be used for renal hydatid disease $[25,74,75]$. The surgical approach is the ultimate treatment with a high cure rate, and most of the patients will be completely cured; however, surgery cannot entirely prevent a recurrence. The prescription of albendazole for six months after cystectomy is suggested for the prevention of recurrence. Moreover, albendazole is recommended for patients with liver or lung CE that are not suitable for surgery, for patients with multiple cysts in different organs, and/or peritoneal hydatid cysts [25].

In hepatic $\mathrm{CE}$, surgery is only suggested for the management of CE2 and CE3, while chemotherapy and percutaneous treatment are suggested for CE1, CE2, and $\mathrm{CE} 3 \mathrm{a} / \mathrm{CE} 3 \mathrm{~b}$. The cysts in CE4 and CE5 stages are inactive; no treatment is required as long as there are no complications, and the preferred strategy is a "watch and wait" approach [15].

\section{Concluding remarks}

Renal echinococcosis is a rare disease, yet it can be serious. Very little information is available about genotypes of the parasites causing renal CE. More work on the immunology of renal echinococcosis is recommended. Its diagnosis is complicated and cannot be based solely on immunological techniques. However, imaging techniques are very valuable for the diagnosis of the disease. The treatment is mostly based on conservative or radical cystectomy; radical nephrectomy should be avoided if the renal parenchyma is not destroyed.

\section{References}

1. Zhang W, Wen H, Li J, Lin R, McManus DP (2012) Immunology and immunodiagnosis of cystic echinococcosis: an update. Clin Dev Immunol 2012: 101895.

2. Pakala T, Molina M, Wu GY (2016) Hepatic echinococcal cysts: A review. J Clin Transl Hepatol 4: 39-46.

3. Agudelo Higuita NI, Brunetti E, McCloskey C (2016) Cystic echinococcosis. J Clin Microbiol 54: 518-523.

4. Thompson RC (2017) Biology and systematics of echinococcus. Adv Parasitol 95: 65-109.

5. Singh BB, Dhand NK, Ghatak S, Gill JP (2014) Economic losses due to cystic echinococcosis in India: Need for urgent action to control the disease. Prev Vet Med 113: 1-12.

6. Ammann RW, Eckert J (1996) Cestodes. Echinococcus. Gastroenterol Clin North Am 25: 655-689.

7. Wen H, Vuitton L, Tuxun T, Li J, Vuitton DA, Zhang W, McManus DP (2019) Echinococcosis: advances in the 21st Century. Clin Microbiol Rev 32: e75-18
8. Craig PS, Hegglin D, Lightowlers MW, Torgerson PR, Wang Q (2017) Echinococcosis: control and prevention. Adv Parasitol 96: 55-158.

9. Jenkins DJ, Lievaart JJ, Boufana B, Lett WS, Bradshaw H, Armua-Fernandez MT (2014) Echinococcus granulosus and other intestinal helminths: current status of prevalence and management in rural dogs of eastern Australia. Aust Vet J 92: 292-298

10. Torgerson PR, Keller K, Magnotta M, Ragland N (2010) The global burden of alveolar echinococcosis. PLoS Negl Trop Dis 4: e722.

11. Deplazes P, Rinaldi L, Alvarez Rojas CA, Torgerson PR, Harandi MF, Romig T, Antolova D, Schurer JM, Lahmar S, Cringoli G, Magambo J, Thompson RC, Jenkins EJ (2017) Global distribution of alveolar and cystic echinococcosis. Adv Parasitol 95: 315-493.

12. Vuitton DA, Demonmerot F, Knapp J, Richou C, Grenouillet F, Chauchet A, Vuitton L, Bresson-Hadni S, Millon L (2015) Clinical epidemiology of human AE in Europe. Vet Parasitol 213: $110-120$

13. Gottstein B, Stojkovic M, Vuitton DA, Millon L, Marcinkute A, Deplazes P (2015) Threat of alveolar echinococcosis to public health--a challenge for Europe. Trends Parasitol 31: 407-412.

14. Harris A, Heath DD, Lawrence SB, Shaw RJ (1989) Echinococcus granulosus: ultrastructure of epithelial changes during the first 8 days of metacestode development in vitro. Int J Parasitol 19: 621-629.

15. Brunetti E, Kern P, Vuitton DA (2010) Expert consensus for the diagnosis and treatment of cystic and alveolar echinococcosis in humans. Acta Trop 114: 1-16.

16. Tekin R, Onat S, Tekin RC (2016) Hydatid cysts in a patient with multiple organ involvement. Rev Soc Bras Med Trop 49: 534.

17. Larrieu EJ, Frider B (2001) Human cystic echinococcosis: contributions to the natural history of the disease. Ann Trop Med Parasitol 95: 679-687.

18. Tekin R, Kara AF, Tekin RC, Cimen D (2011) Cardiac hydatid cyst case recovered with medical treatment. Anadolu Kardiyol Derg 11: 650-651.

19. Tekin R, Avci A, Tekin RC, Gem M, Cevik R (2015) Hydatid cysts in muscles: clinical manifestations, diagnosis, and management of this atypical presentation. Rev Soc Bras Med Trop 48: 594-598.

20. Grosso G, Gruttadauria S, Biondi A, Marventano S, Mistretta A (2012) Worldwide epidemiology of liver hydatidosis including the Mediterranean area. World J Gastroenterol 18: 1425-1437.

21. Rokni MB (2009) Echinococcosis /hydatidosis in Iran. Iranian J Parasitol 4: 1-16.

22. Zmerli S, Ayed M, Horchani A, Chami I, El Ouakdi M, Ben Slama MR (2001) Hydatid cyst of the kidney: diagnosis and treatment. World J Surg 25: 68-74.

23. Agarwal MM, Hemal AK (2011) Surgical management of renal cystic disease. Curr Urol Rep 12: 3-10.

24. Gupta A, Gupta J, Devkaran B, Gupta A (2017) Primary renal echinococcosis with gross hydatiduria. BMJ Case Rep 2017: bcr-2017-220502

25. Rexiati M, Mutalifu A, Azhati B, Wang W, Yang H, Sheyhedin I, Wang Y (2014) Diagnosis and surgical treatment of renal hydatid disease: a retrospective analysis of 30 cases. PLoS One 9: e96602. 
26. Jafari R, Sanei B, Baradaran A, Spotin A, Bagherpour B, Darani HY (2017) Genetic characterization of Echinococcus granulosus strains isolated from humans based on nad1 and cox1 gene analysis in Isfahan, central Iran. J Helminthol: 1-7.

27. Sadjjadi SM, Mikaeili F, Karamian M, Maraghi S, Sadjjadi FS, Shariat-Torbaghan S, Kia EB (2013) Evidence that the Echinococcus granulosus G6 genotype has an affinity for the brain in humans. Int J Parasitol 43: 875-877.

28. Schneider R, Gollackner B, Schindl M, Tucek G, Auer H (2010) Echinococcus canadensis G7 (pig strain): an underestimated cause of cystic echinococcosis in Austria. Am J Trop Med Hyg 82: 871-874.

29. Hizem A, M'Rad S, Oudni-M'rad M, Mestiri S, Hammedi F, Mezhoud H, Zakhama A, Mokni M, Babba H (2016) Molecular genotyping of Echinococcus granulosus using formalin-fixed paraffin-embedded preparations from human isolates in unusual tissue sites. J Helminthol 90: 417-421.

30. Zhang W, You H, Li J, Zhang Z, Turson G, Aili H, Wang J, McManus DP (2003) Immunoglobulin profiles in a murine intermediate host model of resistance for Echinococcus granulosus infection. Parasite Immunol 25: 161-168.

31. Dempster RP, Harrison GB, Berridge MV, Heath DD (1992) Echinococcus granulosus: use of an intermediate host mouse model to evaluate sources of protective antigens and a role for antibody in the immune response. Int J Parasitol 22: 435-441.

32. Rogan MT, Craig PS, Zehyle E, Masinde G, Wen H, Zhou P (1992) In vitro killing of taeniid oncospheres, mediated by human sera from hydatid endemic areas. Acta Trop 51: 291296.

33. Khabiri AR, Bagheri F, Assmar M, Siavashi MR (2006) Analysis of specific IgE and IgG subclass antibodies for diagnosis of Echinococcus granulosus. Parasite Immunol 28: 357-362.

34. Daeki AO, Craig PS, Shambesh MK (2000) IgG-subclass antibody responses and the natural history of hepatic cystic echinococcosis in asymptomatic patients. Ann Trop Med Parasitol 94: 319-328.

35. Sterla S, Sato H, Nieto A (1999) Echinococcus granulosus human infection stimulates low avidity anticarbohydrate IgG2 and high avidity antipeptide IgG4 antibodies. Parasite Immunol 21: 27-34.

36. Sarkari B, Rezaei Z (2015) Immunodiagnosis of human hydatid disease: Where do we stand? World J Methodol 5: 185195.

37. Hadipour M, Nazari M, Sanei B, et al. (2016) Immunological diagnosis of human hydatid cyst using Western immunoblotting technique. J Res Med Sci 21: 130.

38. Gonzalez-Sapienza G, Lorenzo C, Nieto A (2000) Improved immunodiagnosis of cystic hydatid disease by using a synthetic peptide with higher diagnostic value than that of its parent protein, Echinococcus granulosus antigen B. J Clin Microbiol 38: 3979-3983.

39. Lightowlers MW, Liu DY, Haralambous A, Rickard MD (1989) Subunit composition and specificity of the major cyst fluid antigens of Echinococcus granulosus. Mol Biochem Parasitol 37: 171-182.

40. Chookami MB, Sharafi SM, Sefiddashti RR, Jafari R, Bahadoran M, Pestechian N, Yousofi Darani H (2016) Effect of two hydatid cyst antigens on the growth of melanoma cancer in C57/black mice. J Parasit Dis 40: 1170-1173.

41. Yousofi Darani H, Yousefi M, Safari M, Jafari R (2016) Parasites and immunotherapy: with or against? J Parasit Dis 40: 217-226.
42. Yousofi Darani H, Sharafi SM, Mokarian F, Yousefi M, Sharafi SA, Jafari R (2016) Therapeutic effect of hydatid cyst liquid on melanoma tumor growth in mouse model. Br J Med Med Res 18: 1-7.

43. Aref N, Shirzad H, Yousefi M, Yousofi Darani H (2013) Effect of different hydatid cyst molecules on Hela and Vero cell lines growth in vitro. J Immunodefic Disor 2: doi:10.4172/2324853X.1000105

44. Sharafi SM, Rafiei R, Rafiei R, Hadipour M, Shirzad H, Khanahmad H, Yousofi Darani H (2016) A nonglycosylated 27 $\mathrm{kDa}$ molecule as common antigen between human breast cancer and Echinococcus granulosus hydatid cyst wall. Adv Breast Cancer Res 5: 90.

45. Daneshpour S, Bahadoran M, Yousefi M, Mortazavi N, Mahmoudzadeh M, Eskandarian A, Hejazi SH, Yousofi Darani H (2014) Immunological cross reaction between cancer cells and hydatid cyst. J Shahrekord Univ Med Sci 16: 99-105.

46. Daneshpour S, Bahadoran M, Hejazi SH, Eskandarian AA, Mahmoudzadeh M, Darani HY (2016) Common antigens between hydatid cyst and cancers. Adv Biomed Res 5: 9.

47. Gogus C, Safak M, Baltaci S, Turkolmez K (2003) Isolated renal hydatidosis: experience with 20 cases. J Urol 169:186189.

48. Chen X, Zhang J, Feng X, Chen X, Yin S, Wen H, Zheng S (2014) Humoural immune response and pathological analysis in patients with false immune diagnosis of cystic echinococcosis. Parasite Immunol 36: 170-176.

49. Magambo JK, Zeyhle, Wachira TM, Wachira J, Raasen T (1995) Cellular immunity to Echinococcus granulosus cysts. Afr J Health Sci 2: 250-253.

50. Lightowlers MW (2006) Vaccines against cysticercosis and hydatidosis: foundations in taeniid cestode immunology. Parasitol Int 55 Suppl: 39-43.

51. Rogan MT, Bodell AJ, Craig PS (2015) Postencystment/established immunity in cystic echinococcosis: is it really that simple? Parasite Immunol 37: 1-9.

52. Bortoletti G, Gabriele F, Conchedda M (2004) Natural history of cystic echinococcosis in humans. Parassitologia 46: 363366.

53. Brunetti E, Garcia HH, Junghanss $T$ (2011) Cystic echinococcosis: chronic, complex, and still neglected. PLoS Negl Trop Dis 5: e1146.

54. Romagnani S (1991) Type $1 \mathrm{~T}$ helper and type $2 \mathrm{~T}$ helper cells: functions, regulation and role in protection and disease. Int $\mathrm{J}$ Clin Lab Res 21: 152-158.

55. Allen JE, Maizels RM (2011) Diversity and dialogue in immunity to helminths. Nat Rev Immunol 11: 375-388.

56. Diaz A, Casaravilla C, Allen JE, Sim RB, Ferreira AM (2011) Understanding the laminated layer of larval Echinococcus II: immunology. Trends Parasitol 27: 264-273.

57. Tamarozzi F, Mariconti M, Neumayr A, Brunetti E (2016) The intermediate host immune response in cystic echinococcosis. Parasite Immunol 38: 170-181.

58. Dematteis S, Baz A, Rottenberg M, Fernandez C, Orn A, Nieto A (1999) Antibody and Th1/Th2-type responses in BALB/c mice inoculated with live or dead Echinococcus granulosus protoscoleces. Parasite Immunol 21: 19-26.

59. Rostami-Rad S, Jafari R, Yousofi Darani H (2018) Th1/Th2type cytokine profile in C57 black mice inoculated with live Echinococcus granulosus protoscolices. J Infect Public Health 11: 834-839.

60. Dorosti Z, Tolouei S, Khanahmad H, Jafari R, Jafaee F, Sharafi SM, Darani HY (2016) IL-4 gene expression in adventitial 
layer (fibrous layer) of hepatic ovine and bovine hydatid cysts. J Parasit Dis 40: 855-859.

61. Rigano R, Profumo E, Bruschi F, Carulli G, Azzara A, Ioppolo S, Buttari B, Ortona E, Margutti P, Teggi A, Siracusano A (2001) Modulation of human immune response by Echinococcus granulosus antigen B and its possible role in evading host defenses. Infect Immun 69: 288-296.

62. Coban G, Orhan Z, Kalkan S, Tuzun U, Unver N (2016) Renal Hydatid Cyst. Bezmialem Science 4: 33-35.

63. Peng X, Li J, Wu X, et al. (2006) Detection of Osteopontin in the pericyst of human hepatic Echinococcus granulosus. Acta Trop 100: 163-171.

64. Wu XW, Chen XL, Zhang SJ, Zhang X, Sun H, Peng XY (2011) Pericyst may be a new pharmacological and therapeutic target for hydatid disease. Chin Med J 124: 2857-2862.

65. Jafari R, Sanei B, Baradaran A, Kolahdouzan M, Bagherpour B, Yousofi Darani H (2018) Immunohistochemical observation of local inflammatory cell infiltration in the hosttissue reaction site of human hydatid cysts. J Helminthol: 1-9.

66. Sehgal N, Priyadarshi V (2017) Beware! A simple renal cyst could be a hydatid cyst. Urol Ann 9: 281-284.

67. Turgut AT, Odev K, Kabaalioglu A, Bhatt S, Dogra VS (2009) Multitechnique evaluation of renal hydatid disease. AJR Am J Roentgenol 192: 462-467.

68. Wuestenberg J, Gruener B, Oeztuerk S, Mason RA, Haenle MM, Graeter T, Akinli AS, Kern P, Kratzer W (2014) Diagnostics in cystic echinococcosis: serology versus ultrasonography. Turk J Gastroenterol 25: 398-404.

69. Sansot M, Le Treut Y, Burger G, Marlois O, Jouve P (1983) Symptomatology of pseudotumoral forms of hydatid cyst of the liver. Apropos of 7 cases. Ann Radiol 26: 370-375.
70. Beggs I (1985) The radiology of hydatid disease. AJR Am J Roentgenol 145: 639-648.

71. Angulo JC, Sanchez-Chapado M, Diego A, Escribano J, Tamayo JC, Martin L (1997) Renal echinococcosis: clinical study of 34 cases. J Urol 157: 787-794.

72. Goel MC, Agarwal MR, Misra A (1995) Percutaneous drainage of renal hydatid cyst: early results and follow-up. $\mathrm{Br}$ J Urol 75:724-728.

73. Odev K, Kilinc M, Arslan A, Aygun E, Gungor S, Durak AC, Yilmaz K (1996) Renal hydatid cysts and the evaluation of their radiologic images. Eur Urol 30: 40-49.

74. Cretu CM, Codreanu RR, Mastalier B, Popa LG, Cordos I, Beuran M, Ianulle DA, Simion S (2012) Albendazole associated to surgery or minimally invasive procedures for hydatid disease--how much and how long. Chirurgia 107: 1521.

75. Yasawy MI, Mohammed AE, Bassam S, Karawi MA, Shariq S (2011) Percutaneous aspiration and drainage with adjuvant medical therapy for treatment of hepatic hydatid cysts. World J Gastroenterol 17: 646-650.

\section{Corresponding author}

Rasool Jafari

Department of Medical Parasitology and Mycology, School of Medicine, Urmia University of Medical Sciences, Urmia, Iran, Postal Code:571478334

Tel: +984432234897

Fax: +98 4432229059

Email: rasooljafariii@gmail.com; jafari.r@umsu.ac.ir

Conflict of interests: No conflict of interest is declared. 